[Estudios]

\title{
Estudio comparado de la noción deweyana de naturaleza y los términos confucianos «xing》 (性) y «tian» (天)
}

\section{Comparative Study of Dewey's Notion of Nature and Confucius” Terms «Xing» (性) and «Tian» (天)}

\author{
GLORIA LUQUE MOYA \\ Universidad de Málaga (España)
}

recibido: 03.01 .2018

aceptado: 18.06 .2018

\section{RESUMEN}

Este artículo trata de ampliar el enfoque de estudio de la noción deweyana de naturaleza mediante un análisis comparado con la concepción que Confucio propone en las Analectas. Siguiendo la metodología comparada desarrollada por los autores Roger Ames y David Hall, este estudio atiende a las cualidades que el filósofo estadounidense le atribuye a dicha noción en relación con los caracteres chinos xing y tian. El objetivo de esta aproximación no reside en poner de manifiesto similitudes forzadas, sino en enfatizar el contraste de ideas, las diferencias que distinguen los autores comparados. Obviamente, para establecer este diálogo estos filósofos deben compartir algunas ideas comunes y este será el aspecto significativo de esta comparación: subrayar las conexiones entre ambos filósofos, sin olvidar el contexto y los diferentes influjos desde los que estos dos pensadores desarrollan sus propuestas.

PALABRAS CLAVE

ONTOLOGÍA; PRAGMATISMO; EXPERIENCIA

ABSTRACT

This article attempts to broaden the approach to Deweyan notion of nature by means of a comparative analysis with the conception which Confucius proposes in the Analects. Following the comparative methodology developed by the authors Roger Ames and David Hall, this study deals with the qualities which the American philosopher attributes to this notion in relation with the Chinese characters xing and tian.

Claridades. Revista de filosofía 11 (2019), pp. 9-31

ISSN: 1889-6855 ISSN-e: 1989-3787 Dl.: PM 1131-2009

Asociación para la promoción de la Filosofía y la Cultura (FICUM) 
The goal of this approach does not lie in showing forced similarities, but in emphasizing the contrast of ideas, the differences which distinguish the authors compared. Obviously, in order to establish this dialogue these philosophers must share some commonideas, and this will be a significant aspect of the comparison: to highlight the connections between both philosophers, withoutforgetting the context and the different influences from which these two thinkers develop their proposals KEYWORDS

ONTOLOGY; PRAGMATISM; EXPERIENCE

«Sin contorno.

Nada de rodeos.

¡A través!

La vida, la agitada, está en esas travesías en las que, sin embargo, un no sabe cómo orientarse ni cómo volver a ella.

Volver a la danza original de los seres más allá de la forma y de todo el tejido conjuntivo del que está atiborrada, más allá de ese inmóvil embalaje que es la piel. ¿Qué miramos además de lo que miramos, o de menos, o de través?

Danza originales de los seres, tras la cual resultan tolerables». ${ }^{1}$

\section{INTRODUCCIÓN}

LA PUBLICACIÓN DE LA OBRA Experienciay Naturaleza (1925) supone un punto de inflexión en la filosofía de John Dewey, no tanto por inaugurar el periodo final de su pensamiento, sino porque en ella desarrolla una propuesta ontológica que parece devolverlo a las cuestiones metafísicas de las que se ocupó en su etapa inicial. El filósofo estadounidense formula su proyecto ontológico tratando de superar los dos problemas principales que, según él, habían afectado a la filosofía moderna debido a la minusvaloración e incluso olvido de la cotidianeidad. El primero de ellos surgía al intentar establecer una integración entre las creencias del hombre sobre el mundo en el que vive y las creencias sobre los valores y los propósitos que deberían dirigir su conducta ${ }^{2}$. El segundo radicaba en la posición privilegiada que se

1 Michaux, H.: Ideogramas en China. Captar mediante trazos. Madrid: Circulo de Bellas Artes, 2006, p. 85

2 Dewey, J.: Logic. The Theory of Inquiry. The Later Works of John Dewey, vol. 14. Carbondale: Southern Illinois University Press, 2008, p. 8. En adelante las referencias a los escritos de John Dewey se basarán en la edición crítica de las obras completas publicada por Southern Illinois University: EW (The Early Works), MW (The Middle Works) y LW (The Later Works). Las citas seguirán el modelo normalizado entre los estudiosos de la obra de Dewey: la inicial de las series, seguida por el volumen y el número de la página. 
le atribuía a la ciencia física frente a las cosas procedentes de la experiencia ordinaria (L, LW 14:9). Estos dos problemas generaron una serie de dualismos basados en la primacía del objeto de conocimiento frente a lo real, frente a las cosas que no provenían de la experiencia cognitiva.

En oposición a estos dualismos que negaban la cotidianeidad y nuestro entorno más inmediato, el filósofo americano trata de recuperar el valor y el significado de la vida humana mediante su propuesta ontológica, conocida como emergentismo. De este modo, Dewey propone una metafísica que recibe duras críticas ${ }^{3}$ y que, en primera instancia, puede mostrarse contradictoria, si tenemos en cuenta que el propio autor argumenta en contra de lo que podíamos denominar metafísica tradicional o sustancialista.

No obstante, la introducción de esta metafísica naturalista ${ }^{4}$ es necesaria si consideramos la noción clave sobre la que se construye su filosofía, la experiencia. Como ha destacado Carlos Mougán ${ }^{5}$, quedarse en una mera descripción de la experiencia implicaría una forma de escepticismo e incluso de idealismo. Dewey, en cambio, pretende desarrollar una filosofía que atienda a esa relación entre criatura viva y medio, que considere la experiencia en el continuo devenir de la vida.

Siguiendo los estudios que han realizado especialistas de la obra de Dewey, como S. Morris Eames, Larry Hickman, Joseph Margolis y Thomas Alexander, estas páginas analizan la noción clave que el filósofo estadounidense emplea para denominar a dicho medio, «la naturaleza». Para ello, utilizaré una metodología comparada que ponga en relación la noción deweyana con la filosofía de Confucio y los caracteres xing y tian.

Esta aproximación continúa la línea de investigación abierta por los filósofos Roger Ames y David Hall, según los cuales el pragmatismo de Dewey parece compartir con Confucio una preocupación filosófica central sobre cuál es la vía en la que deberíamos desplegar nuestra existencia. Esto es, en contraposición a la tradición occidental que había especulado sobre cuestiones en torno a qué es la verdad y cuál es el ser de las cosas,

3 La crítica más celebre será la que realiza Rorty sobre lo que el autor denomina «la parte oscura de Dewey», «el mal Dewey», cuyo contenido se centra en cuestiones metafísicas: Rorty, R.: Consequences of Pragmatism. Hassocks: Harvester Press, 1982, pp. 73-74. 4 Término empleado por Carlos Mougán en Mougán Rivero, J. C: Acción y racionalidad. Actualidad de la obra de John Dewey. Cádiz: Universidad de Cádiz, 2000, p. 142 y ss. 5 Ibid. 
ambos autores van a plantear preguntas sobre el cómo ${ }^{6}$, van a reflexionar sobre el modo en que desplegar una vida significativa en nuestra continua interacción con la naturaleza.

Dewey va a alejarse, de esta manera, de la tradición filosófica occidental y va a mostrarse más cercano a otras corrientes de pensamiento que no ponen el foco de atención en principios o teorías universales, sino en un modo de acción que promueva una existencia armónica. En este contexto, el análisis sobre el concepto deweyano de naturaleza se ve enriquecido al ponerlo en diálogo con la filosofía de Confucio porque ambos ponen el acento en la mutua interacción y enriquecimiento del hombre y su medio.

Según Ames y Hall ${ }^{7}$, el filósofo chino expone un cosmos, caracterizado por un orden estético que será consecuencia de las actividades de personas específicas en contextos concretos ${ }^{8}$. Es decir, su propuesta ontológica rechaza la existencia de principios universales o patrones impuestos, a favor de una concepción de la realidad que está por desarrollarse en los eventos futuros. Las convergencias que esta propuesta parece mostrar con la noción de naturaleza desarrollada en la ontología deweyana marcarán el análisis comparado que se recoge a continuación. Para ello, formularé una serie de cualidades que caracterizan la noción deweyana en relación con los caracteres que emplea Confucio. A modo de conclusión, apuntaré que ambos autores desarrollan una filosofía del cambio, según la cual la naturaleza es un proceso en continuo hacerse, en el que el hombre participa creativamente. Sin embargo, antes de comenzar es necesario destacar las dificultades que la propia noción de naturaleza plantea.

\section{LÍMITES Y DIFICULTADES DE LA NOCIÓN DE NATURALEZA}

El análisis del término «naturaleza» resulta complejo si tenemos en cuenta que es usado comúnmente en el lenguaje ordinario, así como en el contexto filosófico y académico, de diferentes maneras y con diversos significados. A esto se le suma otra serie de dificultades si se pretende realizar un examen comparado sobre la noción propuesta por el filósofo

6 Hall, D. A. y Ames, R. T.: Thinking from the Han. Self, Truth and Transcendence in Chinese and Western Culture. Albany: State University of New York Press, 1998, pp. 103-104. 7 Hall, D. A. y Ames, R. T.: Thinking through Confucius. Albany: State University of New York Press, 1987, p. 16; Hall, D. A. y Ames, R. T.: op. cit. (1998), p. 111.

8 Hall, D. A. y Ames, R. T.: op. cit. (1987), p. 16. 
estadounidense John Dewey y el pensador chino Confucio.

En primer lugar, las reflexiones sobre la naturaleza en el contexto estadounidense han venido marcadas por el idilio y la seducción que vivieron los «urbanitas» con su entorno natural. Como ha señalado John McDermott ${ }^{9}$, este idilio surgía por la experiencia del vasto territorio estadounidense. La magia del encuentro con la extensión y la grandeza de su medio se puede rastrear en obras como la de Emerson donde leemos:

«El cautivador paisaje que vi esta mañana indudablemente está compuesto por una veintena o treintena de granjas. Miller es propietario de este campo, Locke de ese y Manning del bosque de árboles de más allá. Pero ninguno de ellos posee el paisaje. Hay una propiedad en el horizonte que ningún hombre tiene, excepto aquel cuyo ojo puede integrar todas las partes, es decir, el poetas. ${ }^{10}$

John Dewey, en cambio, aunque se siente cautivado por la grandeza de su entorno natural, no restringe el término «naturaleza» exclusivamente a este entorno, sino que lo define como aquello que hay o existe. Esto es, siguiendo la filosofía pragmatista, la noción naturaleza va a excluir toda idea que implique la existencia de una sustancia, ente o instancia supernatural; la naturaleza no es «esto»o «aquello» sino «lo que se da» ${ }^{11}$.

En segundo lugar, hay que remarcar que Confucio no ofrece una definición explícita de dicho término. Como ha señalado Deborah Sommer ${ }^{12}$, hay dos problemas fundamentales en el análisis de la noción de naturaleza en la filosofía confuciana: por un lado, este término apenas es mencionado en los primeros textos del confucianismo, e incluso los discípulos del filósofo han lamentado que pocas veces oyeron hablar a Confucio sobre la naturaleza; por otro lado, existe una problemática en relación a la traducción de los términos.

En esta propuesta seguiré la línea de investigación abierta por Roger Ames y David Hall y consideraré los caracteres «xing》 (性) y «tian» (天) para

9 Véase McDermott, J.: «Nature Nostalgia and the City», en The Culture of Experience. Philosophical Essays in the American Grain. New York: New York University Press, 1976, pp. 179-204.

10 Emerson, R. W.: Nature. The Essential Writings of Ralph Waldo Emerson. New York: Modern Library, 2000, p. 5.

11 Para un análisis más detallado de las cualidades de la noción de naturaleza en este movimiento filosófico, veáse Ryder J.: «Part I. Conceptions of Nature» en American Philosophic Naturalism in the Twentieth Century. New York: Prometheus Books, 1995.

12 Sommer, D.: «Xing» en Xinzhong Yao (ed.), RoutledgeCurzon Encyclopedia of Confucianism, vol. II, New York: Routledge, 2003, p. 696. 
definir esta noción. El primero de ellos alude a la naturaleza y la naturaleza humana, y en algunos casos se ha usado para denominar a la vida misma ${ }^{13}$. El segundo suele presentarse como una fuerza no personal, un continuo proceso que contiene y genera su propio movimiento mediante la interacción de fuerzas complementarias. En palabras de Ames y Hall, «Toda existencia es un continuo en el que cada aspecto está constituido dentro de un proceso de transformación determinado por su propio impulso y por el conjunto de condiciones que lo posibilitan. $\rangle^{14}$ La complejidad de estos términos requeriría un análisis más profundo que por extensión y complejidad se aleja del objetivo de estas páginas. Por ello, debido al propósito de este estudio, a continuación me limito a considerar dichos términos en relación con las características que Dewey atribuye a la naturaleza.

\section{Principales Características De LA NOCIÓN DEWEYANA DE «NATURALEZA»}

\section{III.1. LA NATURALEZA COMO TOTALIDAD DINÁMICA}

La primera de las cualidades que caracteriza la noción deweyana de naturaleza será aquella que la define como totalidad dinámica. Desde el inicio de su obra el filósofo estadounidense considera la «naturaleza» como un conjunto dinámico y orgánico, idea que hereda del romanticismo alemán. Antes de que el propio Dewey emprendiera su carrera como filósofo, cuando aún daba clases en un instituto de enseñanza secundaria en Burlington, comenzó a leer clásicos de la historia de la filosofía bajo la dirección del profesor T. Morrey. Con este primer contacto, Dewey accedió a la filosofía a través de revistas como Speculative Philosophy, editada por W. T. Harris, estudiante del idealismo y especialmente de Schelling y Hegel. Este influjo inicial, como ha destacado la hija del filósofo, dejará una huella esencial en su filosofía. ${ }^{15}$

Para entender esta aportación al pensamiento deweyano hay que tener en cuenta la noción de «naturaleza» que Schelling introduce, caracterizada

13 Ibid.

14 «All existence is a continuum on which every aspect is constituted within a process of transformation determined by its own impetus and the matrix of conditions that sponsor it» [D. L. Hall y R. T. Ames (1987), op. cit., p. 238].

15 Dewey, J. M.: «Biography of John Dewey,» en P. A. Schilpp (ed.), The Philosophy of John Dewey. New York: Tudor Publishing Co., 1939, p. 17. 
por su dinamismo y unidad. Como Will Dudley ha puesto de manifiesto ${ }^{16}$, Schelling, como la mayoría de los románticos que habían seguido a Ficthe, se separa de él por su desacuerdo respecto a la noción del «mundo natural» y sus relaciones, presentando esta nueva concepción de naturaleza. Fichte, al igual que Kant, definía el mundo como una totalidad mecánica que se subordinaba a los intereses y fines de sujetos conscientes e independientes de la misma. En contraposición, Schelling introduce la idea de naturaleza como un todo dinámico, racionalmente estructurado, de la cual los sujetos conscientes son una parte integral de la misma.

De este modo, Schelling desarrolló una filosofía de la naturaleza, entendida como física especulativa, que se encargaba de sus características esenciales; es decir, se ocupaba de la naturaleza como un todo, no de cosas particulares o productos. Se trataba, pues, de una ciencia infinita, en contraposición al carácter finito de la física atomista y mecanicista. Schelling la considera desde el holismo y el dinamismo, definiéndola como un conjunto orgánico en continuo cambio ${ }^{17}$. Esta caracterización como totalidad dinámica, pese a las variaciones que se irán introduciendo en el avance del pensamiento deweyano, se mantiene hasta el final de su obra.

Sin embargo, la naturaleza entendida como un todo, aclarará Larry Hickman, no hay que interpretarla como mera identificación ${ }^{18}$, sino como una relación de las partes en las que cada una depende de la otra ${ }^{19}$. En esta misma línea, Thomas Alexander ha resaltado que para Dewey la naturaleza es 'en' la experiencia así como la experiencia es 'en' la naturaleza ${ }^{20}$. Para

16 Dudley, W.: Understanding German Idealism. Durham: Acumen, 2007, p. 188.

17 Véase Johnston, J. S.: «German Post-Kantian Idealism and Dewey’s Metaphysics: Mutual Themes», en P. Faurfield, John Dewey and Continental Philosophy. Carbondale: Southern Illinois University Press, 2010, pp. 6-25.

18 Hickman, L.: «John Dewey: His Life and Work», en L. Hickman, S. Neubert (eds.): John Dewey: Between Pragmatism and Constructivisim. New York: Fordham University Press, 2009, p. 20.

19 Ángel Faerna ha señalado en este respecto cómo el escenario metafísico que Dewey presenta en su obra es el de una pluralidad irreductible que tejen una historia abierta que se va construyendo a través de las conexiones simultáneas que lo atraviesan. [Faerna García-Bermejo, A. M.: «De la naturaleza humana a la naturalizacion del hombre», en A. M. Faerna y M. Torrevejano (eds.), Identidad, individuo e bistoria. Valencia: Pre-Textos, 2003, p. 134].

20 Alexander, Th.: John Dewey's Theory of Art, Experience and Nature. The Horizons of Feeling. Albany: State University of New York Press, 1987, p. 97. 
explicar este aspecto relacional habrá que considerar el influjo de Hegel y el hegelianismo de Green y Bradley.

John Ryder se ha ocupado de esta influencia en su obra The Things in Heaven and Earth: An Essay In Pragmatic Naturalism ${ }^{21}$. Según Ryder, Dewey tomó de Hegel el énfasis en la interdependencia de los diferentes elementos, el cual rechazaba la ontología atomista del modernismo. El filósofo norteamericano conservará este influjo hasta el final de su obra, caracterizando a la naturaleza como un todo constitutivo en el que cada elemento depende del otro. ${ }^{22}$

La conjunción de elementos, por tanto, no hay que entenderla como una mera amalgama o acumulación, sino como un conjunto en el que las diferentes partes se relacionan. Esta característica muestra ciertas similitudes con la noción de «cosmos» como entidad dinámica que se le atribuye a Confucio. Tal y como se indica en las Analectas, el cielo (tian, 天) no habla, sino que se da en los procesos naturales y los elementos que lo forman:

«El Maestro dijo: 'Quiero mantenerme en silencio' Zigong respondió: 'Si el Maestro no hablara, ¿qué anotaríamos nosotros, sus pobres discípulos?' El Maestro contestó: '¿Acaso habla el Cielo? Sin embargo, las cuatro estaciones se suceden y las cien criaturas continúan viviendo. ¿Acaso habla el Cielo? $\rangle^{23}$

Esta aproximación a la naturaleza no es exclusiva de Confucio, sino que el filósofo chino comparte las ideas propias de su contexto. La tradición china proponía un modelo del mundo que no era organizado por algún tipo de entidad superior o ente supernatural. Tu Wei-Ming lo define como un proceso orgánico espontáneo de auto-generación de vida, el cual

21 Ryder, J.: op. cit., 2013, p. 79.

22 Hickman, L. A.: Pragmatism as Post-Postmodernism: Lessons from John Dewey. New York: Fordham University Press, 2007, pp. 136-137.

23 Analectas de Confucio, trad. Chang Shiru. Beijing: Casa Editorial de Enseñanza e Investigación de las Lenguas Extranjeras, 2009,17:19, p.231. La obra de Confucio ha sido traducida al español por diferentes autores y editoriales, entre ellas destaca la traducción del chino de A. Hélène Suárez Girard en Kairós (Lun Yu. Reflexionesy enseñanzas. Barcelona: Kairós, 1997) y la de Joaquin Pérez Arroyo en Paidós (Los cuatro libros. Barcelona: Paidós, 2011). La elección de la traducción de Chang Shiru se justifica por varios motivos: en primer lugar, se trata de una edición trilingüe que incluye la versión original en chino clásico, la traducción al chino mandarin o putonghua y la traducción al español; en segundo lugar, la traducción ha sido encargada al filólogo chino Chang Shiru por la Casa Editorial de Enseñanza e Investigación de las Lenguas Extranjeras de Beijing, para ofrecer lo que ellos consideran una versión satisfactoria al español. 
presenta tres características básicas: continuidad, totalidad y dinamismo ${ }^{24}$. Estos aspectos, como veremos a continuación, tendrán eco en la propuesta deweyana de naturaleza.

\section{III.2. LA NATURALEZA COMO ENTORNO DE POSIBILIDADES}

La segunda cualidad clave será el carácter contingente. Joseph Margo$\operatorname{lis}^{25}$ ha enfatizado que uno de los temas principales en Experiencia y naturaleza (1925) fue destacar esta característica. El propio Dewey señala que el descubrimiento de este hecho implica el comienzo de la sabiduría (EN, LW 1:308), alejándose del cartesianismo, del transcendentalismo kantiano, del esencialismo aristotélico y de los desarrollos filosóficos similares que rechazaban esta cualidad. Por ello, dirá Margolis, la metafísica deweyana es la metafísica de la contingencia de la naturaleza. ${ }^{26}$

El filósofo estadounidense caracteriza el mundo como precario y peligroso (EN, LW 1:42), e indica que dicha precariedad propició el surgimiento de la religión con sus ceremonias, ritos, cultos, mitos, magia. Posteriormente, estas variantes se transformarán en la moral, en la ley, en el derecho y en la industria (EN, LW1: 42). En otras palabras, la salvaguarda contra el cambio que, en un primer momento, fue construida desde la magia, se establece después como una ley necesaria y universal que uniformiza lo natural y atribuye una racionalidad inherente al universo $(\mathrm{EN}$, LW1:45).

En este punto el filósofo norteamericano sigue el pragmatismo clásico y se aleja del pensamiento hegeliano. Para Dewey la diversidad de formas que se pueden ir dando en la naturaleza imposibilita su restricción a conceptos establecidos por la filosofía; por ello, en oposición a Hegel, cualquier fenómeno natural siempre superará los logros filosóficos. El filósofo estadounidense, por tanto, rechaza la abstracción de una naturaleza a priori proponiendo una lógica de la experiencia concreta, de crecimiento y de desarrollo, que trasciende la lógica nominalista y será la base desde la cual el hombre desarrollará un orden estético.

$24 \mathrm{Tu}, \mathrm{W} .:$ «The Continuity of Being: Chinese Visions of Nature», en J. Baird Callicott y Roger T. Ames, Nature in Asian Traditions of Thought: Essays in Environmental Philosophy. Albany: SUNY, 1989, p. 69.

25 Margolis, J.: «John Dewey's Metaphysics of Existence», en J. De Groot, Nature in American Philosophy. Washington: Catholic University of American Press, 2004, p. 142. 26 Ibid. 
De manera similar, Roger Ames y David Hall ${ }^{27}$ han subrayado que la tradición confuciana no comparte con la tradición occidental la creencia en un orden del cosmos. Confucio no busca uniformidades sociales ni leyes naturales inmutables, sino que estas irán siendo modificadas debido al carácter contingente de la naturaleza. En palabras de Confucio:

«Zilu dijo: 'Si el soberano de Wei os pidiera que os hicierais cargo del gobierno, ¿qué es lo primero que haríais?'

El Maestro respondió: ‘'Lo que haría seguro es rectificar los nombres!'

Zilu replicó: ‘¿De veras? Maestro, ¿acaso vais a hacer tal descomunal despropósito? ¿Qué sentido tiene eso de rectificar los nombres?’”

El Maestro dijo: 'iQué burdo eres, You! Cuando el caballero ${ }^{28}$ encuentra algo que desconoce, tiene que ser prudente y reservado. Si los nombres no son los correctos, las palabras no se ajustarán a lo que representan. La incoherencia discursiva conduce al fracaso de la empresa. La frustración de la causa impedirá la prosperidad de los sistemas rituales y de la música, que a su vez, afectara al acierto de penas y castigo. Ante la falta de la justicia, el pueblo no sabrá a qué atenerse.

En consecuencia, las palabras que utiliza el caballero, tienen que ser lógicas. La coherencia de las palabras permitirá la viabilidad de las acciones. Por lo tanto el caballero debe cuidar escrupulosamente la corrección de su lenguaje.' ${ }^{29}$

En este sentido, la contingencia no establecerá una alternativa al orden racional, sino que expone cómo las leyes y reglas son patrones externos que los individuos van introduciendo en su continua interacción con el medio. Así, el orden será estético, se construye en las particularidades de cada contexto. Por ello, leemos en las Analectas: «aquél que repasando los conocimientos adquiridos puede aprender algo nuevo llegará a ser un maestro» ${ }^{30}$.

Esta aproximación presenta similitudes con el discurso deweyano; pese a que ambos autores tendrán puntos de partida distintos. Dewey parte de la crítica al orden racional basado en principios abstractos desde los cuales

27 Hall, D. L. y Ames, R. T.: op. cit., 1987, p. 138.

28 En esta traducción los intérpretes han preferido utilizar el término «caballero» en lugar de «hombre superion» para el carácter junzi (君子), tal y como habitualmente presentan otras traducciones como la de Joaquín Pérez Arroyo (Confucio (2002), Los Cuatro Libros, Barcelona: Paidós Orientalia) o la de Roger Ames y Henry Rosemont Jr. (R. Ames y H. Rosemont (ed.) (1998), The Analects of Confucius. A Philosophical Translation, New York: Ballantine Book, 1998).

29 Analectas de Confucio, cit., 13:3, p. 157.

30 Analectas de Confucio, cit., 2:11, p. 15. 
se consideraban las particularidades concretas de los elementos. Por su parte, la base de Confucio será la propia tradición e ideas compartidas de su época que ponían el énfasis en el cambio y caracterizaban lo natural como dinámico. No obstante, podemos sugerir cierta convergencia si atendemos al énfasis puesto en ambos casos en las particularidades que emergen en la vida y que no propiciarán un orden racional, sino estético.

\section{III.3. LA NATURALEZA COMO CONJUNTO DE CAMBIO}

Esto nos conduce a la tercera característica clave de la naturaleza, la noción de «cambio». Para Dewey, nada era definitivo en la naturaleza (EN, LW1:100) y este carácter contingente había que entenderlo desde el cambio. La naturaleza está en un continuo ir y venir, es precaria y, aunque podemos establecer predicciones y controlar algunas de sus singularidades, sólo podemos hacerlo separando la naturaleza, aislándola en el análisis. Este había sido el modo de proceder de la filosofía occidental desde la Grecia clásica. Incluso la filosofía aristotélica, la cual estaba más cerca de los hechos empíricos y fue abiertamente pluralista, finalmente establece una entidad o principio final y permanente ${ }^{31}$. Por ello, Dewey rechazará la noción de «motor inmóvil» como sustancia primera y las cualidades que Aristóteles le adscribe como eterna, inmutable e inmaterial.

En este sentido, la consideración de la terminología confuciana puede ayudarnos a aclarar las características que Dewey le atribuye. Como Ames y Hall ${ }^{32}$ han señalado en relación al uso erróneo del lenguaje trascendente en las interpretaciones sobre el pensamiento de Confucio, Aristóteles recurre al primer motor inmóvil como causa del cambio y los movimientos. En otras palabras, introduce un principio trascendente para poder explicar el mundo natural.

Sin embargo, Confucio no trata de explicar la realidad dinámica, sino que explora los modos de comportamiento y el potencial de las acciones humanas en este continuo devenir. Como leemos en las Analectas, hay que «saber adaptarse a las circunstancias cambiantes.»» Es decir, la noción de

31 Véase Campbell, J.: «Human Nature», Understanding Dewey. Illinois: Open Court, 1995, pp. 25-65, donde explica cómo Dewey retoma la idea del mundo aristotélico en el que no hay rupturas o saltos, en el que nada ocurre en vano, pero finalmente lo abandona al introducir una entidad que permanece.

32 Hall, D. L. y Ames, R. T.: op. cit., 1987, p. 13.

33 Analectas de Confucio, cit., 9:30, p. 105. 
«naturaleza» del pensamiento confuciano se define como un proceso orgánico en el que interactuamos y participamos. Así leemos en los comentarios al Yi Jing atribuidos a Confucio:

«Son el cielo y la tierra los que proporcionan los modelos y los patrones. Es el momento de que cambie y evolucione. Son el sol y la luna los más brillantes. Son la riqueza y la nobleza las calidades más exaltadas. Son los sabios los que preparan las cosas para el uso práctico y los que inventan los instrumentos para el beneficio del mundo.. $)^{34}$

Esta definición muestra convergencias con la filosofía de Dewey, pese a que, como se ha ido destacando, Confucio sigue la tradición china de su época ${ }^{35}$ y el filósofo norteamericano trata de dejar atrás la visión heredada de la naturaleza.

Dewey intenta superar el problema derivado de la tradición filosófica, el cual oponía al continuo fluir de la naturaleza unas formas eternas, ideales y estáticas. Esta oposición, que no es más que una transformación de esa primera superstición o magia del hombre, asume una dualidad jerárquica en la que se enfatiza la superioridad del reino del ser, de lo inmutable, frente al reino inferior e ilusorio de lo fenoménico. Él reúne dentro de esta aproximación sistemas metafísicos tan «dispares» como los de Platón, Demócrito, Tomás de Aquino, Spinoza, Aristóteles, Kant, Descartes, Comte, Haeckel y Eddy (EN, LW1:55). De hecho, deriva de estos sistemas la propia noción de «experiencia absoluta»; esto es, la posibilidad de experimentar la «realidad suprema» (EN, LW1:56).

Por el contrario, para el filósofo estadounidense el pensamiento y la razón son procedimientos intencionales empleados ante la confusión o desequilibrio que provocan las situaciones indeterminadas de la realidad cambiante. Por ello, dichos procedimientos no pueden ser transformados en un antecedente causal de la realidad (EN, LW1: 61). Es decir, la lógica de reflexión no puede convertirse en una ontología absoluta del ser natural. Es más, según el filósofo norteamericano no hay que sorprenderse si se encuentran diferentes caracterizaciones de la naturaleza, incluso aunque puedan llegar a ser opuestas (EN, LW1:66), ya que los eventos naturales son tan variados y complejos que no hay una única forma de aproximación o conocimiento. Vivimos en un universo que no está acabado, sino que constantemente está decidiendo hacía dónde ir y qué hacer (EN, LW 1:68).

34 Yi Jing, Apéndice III, sección I, capitulo 72. Citado por Y. Feng (1923), op. cit., p. 124. 35 Para ampliar esta consideración véase Tu, W.: op. cit., 1989, p. 67. 


\section{III.4. LA NATURALEZA COMO SISTEMA ORGANIZADO}

La cuarta característica de la naturaleza será la organización, aunque ello no implica un final establecido. Para el análisis de esta cualidad habrá que tener presente el influjo de Darwin, ampliamente tratado por diferentes especialistas de la obra deweyana ${ }^{36}$. Es el caso de Thayer, quien ha destacado que el filósofo norteamericano pasa del hegelianismo al darwinismo porque éste le proporcionó los fundamentos necesarios desde los cuales establecer el proceso orgánico de la vida; esto es, la idea de crecimiento y de potencialidad ${ }^{37}$. A través de la noción de «adaptación» $\mathrm{y}$ «crecimiento», el autor establece un orden en la naturaleza sin recurrir a una instancia supernatural, sino a la propia relación del organismo con el medio, el cual vive en él, mediante él, a través de él, con él.

Será en el capítulo 2 de su obra The Influence of Darwinism on Philosophy (1910), titulado «Nature and Its Good: A Conversation», donde el autor exponga la cuestión genuina sobre la naturaleza tras la aportación darwiniana. Dewey critica cómo la filosofía se había perdido en sueños de otro mundo llamado «supernatural» (MW 4:16), un marco espiritual de valores e ideales generales que ante la pregunta sobre las relaciones con la naturaleza acudía a etiquetas y a contenidos fijos. Siguiendo a Darwin, Dewey, en cambio, no cree ni por un momento en algo diferente a la realidad, algo que esté más allá. La naturaleza no es algo que aparece, sino que está sucediendo, y la ciencia es su trascripción.

En esta obra, el filósofo expone por qué, pese a la insistencia aristotélica en la idea de continuidad o cambio, sigue al darwinismo en este punto. Principalmente, esto se debía a que Aristóteles introdujo un principio fijo e inmutable para explicar la naturaleza. En contraposición, la teoría de la evolución aportaba al pensamiento deweyano una nueva aproximación para estudiar la realidad dinámica: la tendencia al cambio del universo como un proceso de continua «evolución», en el que no existe un diseño (como diría Aristóteles) sino una tendencia y un propósito (MW 4:32). Si bien, esa disposición no habrá que entenderla como un final, sino como un momento del proceso.

36 Veáse Perricone, C.: «The Influence of Darwinism on John Dewey's Philosophy of Art», Journal of Speculative Philosophy 20:1, 2006, pp. 20-41.

37 Thayer, H. S.: Meaning and Action: a Critical History of Pragmatism. Indianapolis: BobbsMerrill, 1968, p. 167. 
Esta tendencia al cambio es una concepción característica que encontramos en la tradición china y en el pensamiento confuciano. El propio Dewey lo reconoce en su artículo sobre filosofía china, «Como piensan los chinos» (1922). En este texto, el filósofo estadounidense afirma que esta predisposición al cambio no hay que definirla como mero fatalismo oriental, sino como una de las contribuciones más valiosas que la filosofía China hace a la cultura humana y en concreto a la sociedad occidental caracterizada por la prisa, la impaciencia, el estrés y la ansiedad (MW 13:223). En otras palabras, la propensión al cambio es un aspecto clave no sólo para entender lo natural, sino también para comprender el resto de sus asuntos políticos y sociales. Así dirá Confucio en las Analectas:

«El Maestro dijo: 'Quiero mantenerme en silencio.'

Zigong respondió: 'Si el Maestro no hablara, ¿qué anotaríamos nosotros, sus pobres discípulos?

El Maestro contestó: '¿'Acaso habla el cielo? Sin embargo, las cuatro estaciones se suceden y las cien criaturas continúan viviendo. ¿Acaso habla el Cielo? $\rangle^{38}$

En este texto Confucio explica que el cielo o naturaleza no es nada excepto las cuatro estaciones siguiendo su curso. Como ha expuesto Feng Youlan, se trata de una gran corriente espontánea de vida, un movimiento sucesivo que Confucio denominará dao $0^{39}$. De manera similar, Lewis E. Hahn, en su estudio comparado sobre la filosofía contextualista, en la cual incluye al pragmatismo y a la filosofía china, ha enfatizado que para Confucio la naturaleza transcurre a través de la corriente de vida que se va desplegando a cada instante ${ }^{40}$. Así, en los comentarios al Yi Jing que Feng Youlan le atribuye a Confucio se expone:

«El sucesivo movimiento del Yin (el elemento pasivo, la materia) y del Yang (el elemento activo, la forma) es llamado Dao. Todo lo que lo sigue de éste es bueno. Aquello que resulta de dicho movimiento es lo natural de las cosas. Cuando el benevolente lo percibe, lo llama benevolencia. Cuando el sabio lo percibe, lo llama sabiduría. La gente común, actuando diariamente de acuerdo a éste, aún no es consciente de él. De este modo el Dao, tal y como lo vislumbra el hombre superior, es sólo apreciado por unos pocos. Es manifestado en el amor y es inmanente en la operación. Da a todas las cosas su espontaneidad sin estar inquieto sobre

38 Analectas de Confucio, cit., 17:19, p. 231.

39 Feng, Y.: A Comparative Study of Life Ideals [1923], en Selected Philosophical Writings of Fung Yu-Lan. Beijing: Foreign Languages Press, 1991, pp. 122-123

40 Hahn, L. E.: Enhancing Cultural Interflow Between East and West: Collected Essays in Comparative Philosophy and Culture. Anhui: Thome H. Fang Institute, 1998, pp. 109-110. 
su futuro como los sabios. ¡Cuán perfecta es su virtud! ¡Cómo de grandioso es su hazaña! La rica evolución es su gran logro; la renovación diaria es su mayor virtud. Lo que da la forma es llamado Kien (Yang); lo que imita la forma es llamado Kuen (Yin)... Lo que es inconmensurable en el movimiento es llamado misterio.» ${ }^{41}$

De este modo, la filosofía confuciana se desarrollará en base a esta concepción china de la naturaleza caracterizada por ese devenir organizado. Desde su propio contexto, Dewey también atribuye este carácter de cambio a la naturaleza en Experiencia y naturaleza (1925), en oposición a la filosofía clásica que la consideraba única, eterna y permanente. Es decir, la tradición había rechazado la diversidad y el cambio, a excepción de las referencias que lo reducían a la resolución de combinaciones permanentes. En palabras del filósofo:

«Si la filosofía clásica dice mucho acerca de la unidad y tan poco sobre la diversidad no reconciliada, tanto sobre lo eterno y permanente, y tan poco sobre el cambio (salvo como algo que deba resolverse en combinaciones de lo permanente), tanto sobre la necesidad y tan poco sobre la contingencia, tanto sobre la comprensión universal y tan poco sobre lo particular reacio, bien puede ser porque la ambigüedad y la ambivalencia de la realidad son realmente penetrantes. Dado que estas cosas forman el problema, la solución es más aparente (aunque no más real), en el grado en que cualquiera que sea la estabilidad o la seguridad que el mundo presente, ésta estará fijada y arrestada.» (En, LW 1:46).

La experiencia, explica el filósofo, nos muestra algo más; en oposición a la identificación común de la realidad con lo que es seguro, lo que está acabado, Dewey reivindica su carácter vital. (EN, LW 1:47). Por ello, pese a que existen ciertas repeticiones y regularidades que nos permiten establecer ciertas predicciones y control, ello no debe conducirnos a considerar la naturaleza como una sustancia ordenada, sino como un proceso organizado en el que el cambio no sólo será posible, sino necesario.

\section{III.5. LA NATURALEZA COMO PROCESO EN CONSTRUCCIÓN}

La quinta cualidad de la naturaleza será su carácter utilitario. Este aspecto presenta dificultades debido a las connotaciones que en el lenguaje cotidiano se le atribuyen, así como a las propias inclinaciones de la tradición filosófica, más interesada en lo supernatural, algo más refinado y «con más emoción» (EN, LW1:69). Por ello, para explicar este carácter recurriré a la figura de Emerson y su interpretación sobre la naturaleza. Dewey muestra ciertas afinidades con esta aproximación, pese a que su interés por

41 Yi Jing, Apéndice III sección I, capítulo 24, Citado por Feng, Y.: op. cit., pp. 122-123. 
la misma no fue tan profundo.

El carácter utilitario podemos rastrearlo en el primer capítulo de su ensayo Nature titulado «Commodity», ${ }^{42}$ donde Emerson explica que la naturaleza provee el material en crudo y la energía por la cual construimos, crecemos, comemos. Como señala R.D. Richardson Jr., la noción de «naturaleza» para el autor se define como «el modo en que las cosas son» ${ }^{43}$, a partir del cual creamos nuevas interacciones. Es decir, la función instrumental que le adscribe a la naturaleza no tendrá un sentido negativo o peyorativo. De este modo, la naturaleza no es solo el material, sino también el proceso y el resultado. ${ }^{44}$ Esta concepción de la «naturaleza», que ya la encontramos en poetas románticos como Coleridge, reconoce la distinción entre natura naturans (la naturaleza como un conjunto de fuerzas y procesos activo) y natura naturata (los productos acabados de la naturaleza), poniendo el énfasis en la interrelación entre ambas ${ }^{45}$.

De manera similar, como ha expuesto Larry Hickman, el constructivismo deweyano une estas dos concepciones de la naturaleza ${ }^{46}$. Para Dewey no existe separación funcional entre la naturaleza y la cultura, sino que ambas son fases, una inicial y otra posterior, de la expansión de los significados de la experiencia humana en las diferentes situaciones ${ }^{47}$. Por ello, a diferencia del enfoque que le atribuye Santayana ${ }^{48}$, Dewey no atiende a este término sólo desde el primer plano en el que se despliega el conocimiento, y mucho menos se presenta como un aspecto ignorado o idealizado ${ }^{49}$. El filósofo estadounidense toma de Emerson ${ }^{50}$ la visión dinámica de la natu-

42 Emerson, R. W.: «Comodity», op. cit., pp. 7-9.

43 Richardson Jr., R. D.: «Emerson and Nature», en J. Porte, S. Morris, The Cambridge Companion to Ralph Waldo Emerson. Cambridge: Cambridge University Press, 1999, p. 97. 44 Ibid., p. 7.

45 Richardson Jr., R. D.: op. cit., p. 98.

46 Hickman, L. A.: op. cit., 2007, p. 139.

47 Ibid.

48 Santayana, G.: «Dewey's Naturalistic Metaphysic», en J. A. Boydston (ed.), The Later Works of John Dewey, vol. 3. Carbondale: Southern Illinois University Press, p. 369. Publicado originalmente en The Journal of Philosophy 22, 1925, pp. 673-688.

49 Hickman, L. A.: op. cit., 2007, p. 139.

50 En palabras de Emerson: «Nada es seguro excepto la vida, la transición, el espíritu energizante. Ningún amor que esté regido por un juramento o un pacto puede estar asegurado contra un amor superior. Ninguna verdad es tan sublime que no pueda convertirse en algo trivial mañana a la luz de nuevos pensamientos. La gente quiere estar 
raleza entendida como proceso, transición, en la que el hombre participa, forma parte y se sirve de ella.

Dewey desarrolla, pues, una ontología basada en esa interacción entre hombre y naturaleza, destacando la polaridad de estos dos elementos; es decir, la interdependencia de ambos, hombre y naturaleza, en las relaciones que constituyen las experiencias. En este sentido, la historia del universo puede verse como un continuo, en el que no hay escisión del tipo naturaleza y hombre o mundo natural y mundo cultural. De ahí que el carácter chino xing (姓) signifique al mismo tiempo naturaleza y naturaleza humana. Esto es, se trata de dónde venimos, del corazón de la que se nace (tal y como muestra la disposición del carácter, compuesto por el carácter xin (心), el corazón; y el carácter sheng (生), que se traduce como nacer.

Esta última cualidad de la naturaleza puede compararse con la exposición que Feng Youlan realiza sobre la filosofía confuciana, definiendo la historia del universo como un todo continuado ${ }^{51}$. Según Feng, para Confucio no hay una demarcación entre el estado de la naturaleza y el estado del arte sino que ambos son un continuo. ${ }^{52} \mathrm{El}$ autor cita los comentarios del Yi Jing que atribuye a Confucio para exponer esta idea de utilidad atribuida a la naturaleza:

«Para el universo, lo más esencial es la vida. Para los sabios, lo más importante es el estado. Lo que mantiene el estado es el amor. Lo que mantiene a la gente es la riqueza. La producción de riqueza, la educación de la gente, y la prohibición de hacer lo que está mal se llama justicia.. ${ }^{53}$

Este texto pone de manifiesto cómo la naturaleza es vida y en ella se dan diferentes elementos para mejorarla como el estado, la riqueza o la educación. El arte es natural, en cuanto que ayuda a la naturaleza, la continua. Para el filósofo chino, por tanto, todos los tipos de arte son útiles para la gente y benefician al mundo. Esta aproximación presenta sugerentes resonancias con la propuesta deweyana, ya que el filósofo estadounidense también trata de mostrar el carácter continuado entre la naturaleza y la cultura.

establecida; sin embargo, sólo en la medida en que están aún por hacerse, hay alguna esperanza para ellos.» [Emerson, R. W., «Circles», en The Selected Writings of Ralph Waldo Emerson, cit., 1841, p. 261].

51 Feng, Y.: op. cit., p. 122.

52 Ibid., p. 123.

53 Yi Jing, Apéndice IV, sección II, capítulo 10, Citado por Feng, Y.: op.cit., p. 123. 
No obstante, Dewey elabora su propuesta en oposición a la filosofía occidental, la cual había caracterizado las dificultades que el hombre encontraba como un mero problema económico o como un problema teórico sobre su adecuación al bien trascendental. Históricamente, las dificultades que la criatura viva experimentaba en la interacción con su medio se habían tratado de manera práctica, como un asunto sobre explotación de recursos; o de manera abstracta, ocupándose del posible conflicto que podía generar a la inteligencia ciertas condiciones sociales.

Esta última aproximación era característica del pensamiento platónico, el cual tomaba los finales que el hombre había ido instaurando como esencias de las cosas, independientemente de la experiencia humana. Es decir, consideraba los significados que el hombre creaba como esencias inmutables, ya que no eran estados mentales y se mostraban independientes de las sensaciones inmediatas y de la imaginería de las cosas físicas. La tradición asumía que dichas significaciones eran algo singular estudiado por una metafísica o lógica que las separaba de la naturaleza (LW 1: 151-153). Sin embargo, como apunta Dewey, el significado no puede existir si no se da esa interacción de los seres humanos con el mundo.

Será desde esta ontología emergentista, basada en la interacción hombre-naturaleza, desde la que habrá que entender el carácter instrumental de la naturaleza. Por ello, en oposición a la opinión de Santayana ${ }^{54}$, quien señala que la filosofía deweyana en este punto trata de justificar todos los aspectos de la cultura norteamericana, Dewey está reivindicando la dimensión creativa del ser humano desde la continuidad hombre-naturaleza. En otras palabras, el carácter utilitario no trata la naturaleza como un aspecto relacional o transicional que disuelve al individuo y su medio en funciones sociales $^{55}$, sino que pone el acento en el continuo proceso de interacción.

Como hemos ido viendo a lo largo de estas páginas, la naturaleza no es un aspecto aislado, ni el mero medio desde el que desarrollar el conocimiento, sino un elemento clave en la filosofía de Dewey. A diferencia de lo que creen filósofos como Santayana, el proyecto filosófico de Dewey no se restringe y limita a su teoría del conocimiento y esto se debe a dos motivos principales. En primer lugar, para Dewey no hay que dejarse embaucar por las comúnmente denominadas «relaciones objetivas», porque la ciencia no

54 Santayana, G.: op. cit., p. 369.

55 Ibid., p. 370. 
es más que otro modo de conocimiento, pero no el único ni el universal (MW 4:17). En segundo lugar, Dewey no cualifica como meramente cognoscitiva la relación con la naturaleza, sino que el filósofo destaca que hay otro tipo de experiencias, cargadas de significado. Así, la naturaleza es utilitaria en cuanto será el material en crudo desde el cual el hombre podrá desarrollar experiencias consumadas.

No obstante, esta aproximación sigue planteando dificultades en nuestra actualidad. Según Roger Ames y David Hall, ${ }^{56}$ pese a que Dewey, al igual que James, fue uno de los pragmatistas que tuvo como cometido extender la comprensión del instrumentalismo más allá de los confines en que lo encerraban los razonamientos técnicos, finalmente fracasa por su sesgo naturalista. Sin embargo, esta problemática se desvirtúa si retomamos la noción de «naturaleza» que Larry Hickman proponía, entendida como esa continuidad entre naturaleza como naturaleza y naturaleza como cultura.

\section{CONCLUSIÓN}

A través del análisis comparado del concepto deweyano de naturaleza de estas páginas, se ha tratado de evidenciar cómo el filósofo estadounidense va a proponer una nueva ontología caracterizada por la continuidad entre hombre y naturaleza en la inmediatez de las situaciones, rechazando así posiciones realistas e idealistas. Estas teorías intelectualistas reducían todo, según Dewey, a un modo de conocimiento que no era fiel a la realidad y que introducía una serie de dualismos y distinciones que habían llevado a la filosofía y la psicología a las más desastrosas consecuencias. En cambio, el filósofo adopta una metafísica emergentista que, como ha destacado Carlos Mougán ${ }^{57}$, posibilitará la continuidad entre naturaleza y experiencia.

Esto es, Dewey desarrolla una ontología que pone el acento en las situaciones, en esos eventos en los que naturaleza y experiencia emergen en un continuo fluir ${ }^{58}$. Por ello, su propuesta ontológica puede actualizarse desde el diálogo con la filosofía confuciana, entendida, en palabras

56 Hall, D. L. y Ames, R. T.: op. cit., 1987, p. 79.

57 Mougán Rivero, J. C.: op. cit., p. 150.

58 Savery, W.: «The Significance of Dewey's Philosophy», en: P.A. Schilpp (ed.), op. cit., pp. $479-513$. 
de Ames y Hall ${ }^{59}$, como una ontología de los eventos. De acuerdo con estos autores, Confucio no desarrolla una filosofía en torno a las cualidades, atributos o características de una instancia sobrenatural, sino que la comprensión de los eventos tiene que darse en las actividades de personas específicas en contextos particulares ${ }^{60}$. Se trata, como ha puesto de relieve Lewis Hahn de una filosofía contextualista, una filosofía del cambio, según la cual la naturaleza es un proceso vivo, dinámico; un proceso que está continuamente forjándose, en el que el hombre participa creativamente. En este sentido podemos concluir con las palabras sobre la tierra del libro clásico de la tradición china, y principal obra de referencia de la filosofía de Confucio, el Yi Jing:

Kunvin: Tierra que recibe la acción del principio activo y que sustenta, produce; hembra que sustenta y produce, concurriendo en la serie de los cuatro actos de la vida de los seres. El grande, en lo que hace, no debe prevenir la acción de las fuerzas naturales; si lo hace fracasará; si actúa después y la sigue, tendrá éxito. Si pone su propio provecho por encima de todo podrá adquirir amigos por un lado, pero los perderá por otro. Si busca la paz, la concordia de todos los poderes, tendrá éxito y será feliz. ${ }^{61}$

\section{REFERENCIAS BIBLIOGRÁFICAS:}

Analectas de Confucio. Trad. Chang Shiru. Beijing: Casa Editorial de Enseñanza e Investigación de las Lenguas Extranjeras, 2009.

Alexander, Th.: John Dewey's Theory of Art, Experience and Nature. The Horizons of Feeling, Albany: State University of New York Press, 1987.

Campbell, J.: «Human Nature», Understanding Dewey, Illinois: Open Court, 1995, pp. 25-65.

Dewey, J.: «Nature and Its Good: A Conversation», en J. A. Boydston (ed.), The Middle Works of John Dewey, vol. 4. Carbondale: Southern Illinois University Press, 1910, pp. 15-30.

Dewey, J.: «As The Chinese Think», en J. A. Boydston (ed.), The Middle Works of John Dewey, vol. 13. Carbondale: Southern Illinois University Press, 1922, pp. 217-227.

59 Hall, D. L. y Ames, R. T.: op. cit., 1987, p. 15.

60 Ibid.

61 Yi King. Libro de las mutaciones. Trad de Charles de Harlez. Palma de Mallorca: José J. Olañeta editor, 2007, p. 57. 
Dewey, J.: Experience and Nature [1925], en J. A. Boydston (ed.), The Later Works of John Dewey, vol. 1. Carbondale: Southern Illinois University Press, 2008.

Dewey, J.: Logic. The Theory of Inquiry [1939], en J. A. Boydston (ed.), The Later Works of John Dewey, vol. 14. Carbondale: Southern Illinois University Press, 2008.

Dudley, W.: Understanding German Idealism. Durham: Acumen, 2007.

Emerson, R. W.: Nature. The Essential Writings of Ralph Waldo Emerson. New York: Modern Library, 2000.

Faerna García-Bermejo, A. M.: «De la naturaleza humana a la naturalizacion del hombre», en A. M. Faerna y M. Torrevejano (eds.), Identidad, individuo e historia. Valencia: Pre-Textos, 2003, pp. 113-139.

Faurfield, P.: John Dewey and Continental Philosophy. Carbondale: Southern Illinois University Press, 2010.

Feng, Y.: Selected Philosophical Writings of Fung Yu-Lan. Beijing: Foreign Languages Press, 1991.

Hall, D. A. y Ames, R. T.: Thinking through Confucius. Albany: State University of New York Press, 1987

Hall, D. A. y Ames, R. T.: Thinking from the Han. Self, Truth and Transcendence in Chinese and Western Culture. Albany: State University of New York Press, 1998.

Hahn, L. E.: Enhancing Cultural Interflow Between East and West: Collected Essays in Comparative Philosophy and Culture. Anhui: Thome H. Fang Institute, 1998.

Hickman, L. A.: Pragmatism as Post-Postmodernism: Lessons from John Dewey. New York: Fordham University Press, 2007.

Hickman, L. y Neubert, S. (eds.): John Dewey: Between Pragmatism and Constructivisim. New York: Fordham University Press, 2009.

Margolis, J.: «John Dewey's Metaphysics of Existence», en J. De Groot, Nature in American Philosophy. Washington: Catholic University of American Press, 2004, pp. 138-156.

McDermott, J.: «Nature Nostalgia and the City», en The Culture of Experience. Philosophical Essays in the American Grain. New York: New York University Press, 1976, pp. 179-204.

Mougán Rivero, J. C: Acción y racionalidad. Actualidad de la obra de John Dewey. Cádiz: Universidad de Cádiz, 2000. 
Perricone, C.: «The Influence of Darwinism on John Dewey's Philosophy of Art», Journal of Speculative Philosophy 20:1, 2006, pp. 20-41.

Richardson Jr., R. D.: «Emerson and Nature», en J. Porte, S. Morris, The Cambridge Companion to Ralph Waldo Emerson. Cambridge: Cambridge University Press, 1999, pp. 97-105.

Rorty, R.: Consequences of Pragmatism. Hassocks: Harvester Press, 1982.

Ryder J.: American Philosophic Naturalism in the Twentieth Century. New York: Prometheus Books, 1995.

Santayana, G.: «Dewey’s Naturalistic Metaphysic» [1925], en J. A. Boydston (ed.), The Later Works of John Dewey, vol. 3. Carbondale: Southern Illinois University Press, pp. 367-384.

Schilpp, P.A. (ed.): The Philosophy of John Dewey. New York: Tudor Publishing Co., 1939.

Thayer, H. S.: Meaning and Action: a Critical History of Pragmatism. Indianapolis: Bobbs-Merrill, 1968.

Tu, W.: «The Continuity of Being: Chinese Visions of Nature», en J. Baird Callicott y Roger T. Ames, Nature in Asian Traditions of Thought: Essays in Environmental Philosophy. Albany: State University of New York Press, 1989.

Xinzhong Yao (ed.), RoutledgeCurzon Encyclopedia of Confucianism, New York: Routledge, 2003.

Yi King. Libro de las mutaciones. Trad de Charles de Harlez. Palma de Mallorca: José J. Olañeta editor, 2007, p. 57.

Gloria Luque Moya es investigadora posdoctoral en el departamento de filosofía de la Universidad de Málaga.

\section{Lineas de investigación:}

John Dewey, Filosofía Comparada, Estética de lo cotidiano.

\section{Publicaciones recientes:}

- «La noción de criatura viva en el naturalismo cultural de John Dewey», Revista Estudios de Filosofía 59 (Enero-Junio 2019).

- «'Sobre mi cadáver'. Un análisis sobre el papel del cuerpo en la obra de Mona Hatoum», Asparkia. Investigación Feminista 33 (2018).

- «El pulso del proceso estético: una ilustración multicultural de la noción deweyana de ritmo», en L. Arenas, R. del Castillo y A. Faerna (Eds.), John Dewey: una estética de este mundo, Zaragoza: Prensas Universitarias de Zaragoza, 2018, 
pp. 367-384. ISBN: 978-84-17358-59-4.

- «Una aproximación a la relación arte vida desde la estética de lo cotidiano», en J.F. Zuñiga (Ed.), Autonomía y valor del arte, Granada: Comares, pp. 103-116. ISBN: 978-84-9045-610-1.

Dirección electrónica: glorialm@uma.es 
